'llu. Revista de Ciencias de las Religiones

ISSN: $1135-4712$

http://dx.doi.org/10.5209/ILUR.57407

\title{
La Declaración 219 de los Textos de las Pirámides como antecedente de la relación Re-Osiris en los Libros del Más Allá del Reino Nuevo
}

\author{
Mariano Bonanno ${ }^{1}$
}

Recibido: 27 de julio de 2016 / Aceptado: 3 de marzo de 2017

Resumen. Los Textos de las Pirámides expresan de modos diferentes los medios por los cuales el faraón lograba el recurso necesario para lograr la transfiguración e integrar el mundo de los dioses. Son numerosas las referencias que relacionan al difunto con Osiris, bien en forma directa (identificación), bien indirectamente. De entre ellas, el contenido de TdP 219 cobra la mayor trascendencia dadas la gran cantidad de alusiones a Osiris y su círculo, el tenor teológico integral de recomposición osiriana, y las referencias a la geografía vinculada a Osiris, entre otras. Este estudio se propone analizar detenidamente TdP 219, enmarcar la relación entre el faraón y Osiris, y abordarla en el contexto de una liturgia mayor. Luego, y a modo de conclusión, se intentará ver de qué modo esta declaración puede ser un antecedente directo de la relación Re-Osiris en los Libros funerarios del Reino Nuevo.

Palabras clave: pirámides; Re; Osiris; regeneración; ritual; liturgia.

\section{[en] The Pyramid Texts Spell 219 as forerunner of the relationship Re-Osiris in the New Kingdom Books of the Netherworld}

\begin{abstract}
The corpus of Pyramids Texts expresses in different ways the means by which the pharaoh achieved the transfiguration and integrated himself into the world of the gods. There are numerous references relating the deceased with Osiris, in direct form or indirectly. Among them, the contents of PT 219 reach the most significant degree given the large number of references to Osiris and his circle, integral theological tenor of osirian recomposition, and the references to geography related to Osiris, among others. This study intends to analyze carefully the PT 219 , framing the relationship between the Pharaoh and Osiris in the context of a larger liturgy. Then, and as conclusion, I seek to explain how this declaration can be a direct antecedent of the relationship of Re and Osiris in the New Kingdom Books of the Netherworld.
\end{abstract}

Keywords: pyramids; Re; Osiris; regeneration; ritual; liturgy.

Sumario. 1. Introducción general. 2. TdP 219 (\$167a-§193c). 3. TdP 219 como antecedente de los Libros del Más Allá del Reino Nuevo. 4. Conclusiones. 5. Referencias bibliográficas.

Cómo citar: Bonanno, M. (2017) La Declaración 219 de los Textos de las Pirámides como antecedente de la relación Re-Osiris en los Libros del Más Allá del Reino Nuevo, en Ilu. Revista de Ciencias de las Religiones 22, 29-51.

\footnotetext{
1 Universidad de Buenos Aires (Argentina).

E-mail: mbonanno1971@gmail.com
} 


\section{Introducción general}

La declaración 219 de los Textos de las Pirámides $-\mathrm{TdP}^{2}$ - en la que el rey se identifica con Osiris, ha sido denominada como «texto sacerdotal» por Hays ${ }^{3}$ y como «liturgia mortuoria» por Assmann 4 . En este sentido, la recitación de la declaración por parte del sacerdote oficiante se genera en un ámbito cúltico con objetivo propiciatorio $^{5}$. Usualmente se utilizan por este motivo la segunda persona -en menor medida la tercera- dado que se recita para un beneficiario mientras permanece silente ${ }^{6}$.

De su estudio de las liturgias mortuorias, textos de transfiguración o s3hw, Assmann concluye que su investigación revela una liturgia adicional, la cual sin embargo, está atestiguada muy frecuentemente y aparece en el mismo orden ya en los TdP: declaraciones 213-219 y 220-222. Asimismo, afirma que en este grupo podemos reconocer la más antigua liturgia mortuoria ${ }^{7}$. Según Hays esta declaración pertenece al Grupo B. Transfiguración, a. Identificación, siendo la declaración más larga, con muy por encima de 700 palabras ${ }^{8}$; asimismo es la segunda y tercera personas las que predominan ${ }^{9}$. La secuencia ${ }^{10}$ completa de esta liturgia ${ }^{11}$ está relacionada con la asunción de espacios, estados y de la autoridad por parte del Rey,

...Provisión de alimentos, incienso y perfume

213 El Rey asciende al cielo

214 El Rey asciende al cielo

215 El Rey asciende al cielo como una estrella

216 El Rey, como una estrella, se desvanece al amanecer con las otras estrellas

217 El rey se une al dios-sol ${ }^{12}$

218 El Rey asume autoridad en el Más Allá

2 Con TdP se alude al número de declaración, mientras que con Pir. se hace alusión a las líneas según la nomenclatura de Sethe 1908-1922.

3 Hays 2012, cita 818.

4 Assmann 1986 y 1990 . Hays afirma que son textos relativos a la «assimilation of the sun god», Hays 2012, tabla $\mathrm{B}, 679$, dentro de la categoría de los que denomina «transfiguration texts» y fueron nombrados inicialmente «departure texts» por Hays 2009a, 54, Spruchfolge A por Altenmüller 1972, 46-47, Gruppe C por Osing 1986, 138-140 y Sequence E1 por Allen 1994, 16-17; ver Hays 2009a, 54, cita 46.

5 «They consistently refer to the deceased in the grammatical second or third person, indicating that these were performed by priests speaking to and about the deceased for his benefit», Hays 2009, 208.

6 Hays 2012, 11. Para los motivos para el cambio de persona o la transformación del agente (que ejecuta el ritual), véase Morales 2016.

7 Assmann 1990, 14.

8 Hays 2012, 273.

9 Para una sinopsis completa de esta declaración, ver Hays 2012, 350.

10 Según Hays, «a sequence is a series of texts which is found on at least two sources having the same component texts in the same order», Hays 2012, 453.

11 «The liturgies figure prominently on the walls of the sarcophagus chamber (if extant) or on the outer coffin, while the "literature" is situated inside, in direct contact with the deceased», Assmann 1990, 14.

12 «The utterance consists of four parts in each of which the king announces his arrival in the sky to the sun-god and commands certain gods, associated with the four cardinal points to broadcast his coming to the four sides of the universe. The symmetry of the composition is heightened by repetitions and relieved by variations»", Lichtheim 1975, 32. 
219 El rey es identificado con Osiris

220 Apertura de la capilla

221 Oración del Rey Coronación del Rey ${ }^{13}$

222 El Rey se une al dios-sol (diálogo entre el sacerdote y el Rey) $)^{14}$

...Provisión de alimentos y conjuros contra criaturas nocivas

La posesión de esta liturgia desencadenaba la continuidad de la transición como 3 , iluminado, «espíritu transfigurado», que completaba o complementaba, los rituales funerarios terrenos que comenzaban el proceso de «espiritualización» del difunto $-s 3 h w^{15}$-. Lo que provocaba este tipo de rituales en que el rey salía del horizonte «espiritualizado» ${ }^{16}$ era parte del progreso que, desde la tumba, iniciaba el proceso de despojamiento de todo «vínculo carnal».

La importancia de esta liturgia reside en la carga que comportaba para la re(constitución) de la investidura rea ${ }^{17}$. En este sentido, la apropiación de un texto originalmente declamativo del poder real en la muerte, implicaba la apropiación de las prerrogativas que el texto conlleva.

La significación de la estrecha relación del faraón con Orión, con la pequeña y gran Enéada, su vinculación con la Duat que lo asimilaba a las estrellas, la(re) asunción del poder que la presentación de las coronas conllevaba, reconfirmaban su ascendencia entre los dioses, razón por la cual se lo concebía como imperecedero.

A propósito de la liturgia de transfiguración en el culto de Osiris en Abidos, Assmann afirma acertadamente que «the situation of these $s 3 h w$ is rather paradoxical. There can be no doubt that they are temple liturgies and not mortuary spells (...). It is, however, equally evident that these temple liturgies are in fact collections of typical mortuary spells. Many of the spells are known as pyramid texts, some as coffin texts, the rest being spells of a similar form and content. The title of these liturgies, s3hw w points rather to the cult of the dead than to that of the gods. $3 h$ is the term for the "transfigured spirit", i.e. the ritually equipped $\gg$ ".

13 Ritual a la corona del Bajo Egipto compuesto por la alocución de un oficiante religioso, una oración pronunciada por el rey y la respuesta de la corona. Véase Goebs 2008, donde analiza este ensalmo a la corona.

14 Todos los miembros del monarca se identifican con una divinidad, y él mismo es Atum para cada dios (TdP 215); es Neftys quien se le aparece como barca de la noche (TdP 216); por último, toma posesión de las coronas como símbolos de su poder real que se extiende también al Más Allá (220-222). Piankoff 1968, 64 afirma que "As a new Osiris, the dead king stands before Atum, the All. The gods of the Ennead, the procreation of Atum acknowledge his authority. He is identified with several local gods and receives a food offering personified as his daughter».

15 O «declaraciones transfigurativas»-verklärenden Sprechen-, Wb IV 24; Assmann 1986,998-999.

16 Así por ejemplo TdP $217 \S 154 \mathrm{~d} w b n=t n 3 h t m b w 3 h n=t n$ im «que puedas elevarte en el horizonte, en el lugar que te sea favorable».

17 Estas prerrogativas y sus efectos se extienden luego a todo difunto que contase con estos textos en el Reino Medio o posteriormente, fuera del contexto de la realeza, en el contexto privado, aunque sin omitir la relación entre la «coronación (regia o no)» y el éxito del difunto en su integración en el mundo de los dioses; véase Morales 2013.

18 Assmann 1990, 4. Véase también Quack 2005, 165-185. 


\section{TdP 219 (\$167a-§193c)}

Schott nombra a la declaración como una Letanía ${ }^{19}$. Para Barta el conjunto de los $\mathrm{TdP}$ tiene como objetivo la transfiguración del difunto ${ }^{20}$ por lo que extensivamente lo es TdP 219 en particular.

Altenmuller ${ }^{21}$ sostiene que la serie 213-222 conforma un conjunto de ritos de procesión funeraria. Para Osing ${ }^{22}$, por su parte, TdP 213-222 son textos relativos a la existencia futura del rey.

Según afirma Allen, «the secuence 213-222 -located in the east and south wall of the sarcophagus chamber $=$ Duat ${ }^{23}$ is a Resurrection Ritual group. The ritual begins with three spells (PT 213-215), addressed solely to the King (inert in his sarcophagus $^{24}$ ), continues with three spells (PT 216-218) meant originally to be spoken by the newly revived King, and concludes with six spells (PT 219-222, 245-246), spoken alternatively to and (originally) by the king $\rangle^{25}$.

Hellum, en su análisis del mito en los TdP denomina a estas alusiones en el marco del mito «de ascensión» y concluye que son usados ciertos dispositivos retóricos de paralelismo y yuxtaposición ${ }^{26}$, entre otros.

19 Schott 1945, 42. »Sie sind strophisch gebaut. Die in den Hymnen erschienenen Formelemente, Verdoppelung und Viergliedschema, sind zu streng gegliederten Litaneien von großem Schwung und Umfang erweitert, die den Geist der Zeit der Pyramidenerbauer atmen, der sie entstammen".

20 Barta 1981, 62ss.

$21 \quad$ Altenmuller 1972, 112-173.

22 Ossing 1982, 138-140.

23 Hays puso en cuestión esta teoría cosmográfica, afirmando que ésta encuentra credibilidad en dos artículos seminales, " "the Cosmology of the Pyramid Texts", where a single page presents the cosmographic interpretation of burial chambers as a feature of the pyramids with texts, y en cinco páginas de "Reading a Pyramid", where an identical interpretative schema is nominally restricted to that of Unis», Hays 2009b, 200-201), del mismo modo, sostiene que «while it is definitely the case that the Egyptians conceptualized the tomb in cosmographic and other terms, the theory that there was an articulated symbology for individual subterranean rooms in the pyramids is untenable. It fails because, even if its premise were true, then the cosmographic identities of the two principal chambers are ambiguous: by applying the theory's reasoning to other evidence, we should have to understand that the sarcophagus chamber is not only the Duat but also the Akhet, that the antechamber is not only the Akhet but also the Duat, and that even the above-ground portion of the tomb is the Akhet», Hays 2009b, 217. Finalmente, Morales plantea, ordenando los ensalmos de los TdP por otros criterios diferentes de los geo-cosmográficos, que los espacios vinculados a la Duat como al Akhet podrían combinarse en una primera etapa, para luego diferenciarse, ver Morales 2016, 237-255. El debate excede la temática que aquí se plantea pero es necesario mencionarlo para enriquecer así la base teórica sobre la que descansa la TdP 219. Una relectura de los ensalmos que trascienda la meramente geo/cosmográfica, podría suponer en este caso un nuevo ordenamiento que vincule ambos espacios de transfiguración.

24 «His (of the King) is Osiris, and clearly remains within the sarcophagus as Osiris remains in the Duat...; the sun is regenerated through its union with Osiris in the Duat», Allen 1994, 25.

25 Allen 1994, 16-17. Véase también Allen 2005, 16.

26 Hellum 2014, 126. En la misma página, en la cita 19 y en referencia al paralelismo, afirma que «in PT 219 §§ $167-178$, found in every kingly Saqqara pyramid, provides a particularly lengthy example, with several different syntactic kinds of parallelism. The first is: 'Atum, this Osiris is your son, who you caused to be restored so that he may live. If he lives, so does this Pepy Neferkare live. If he does not die, this Pepy Neferkare does not die. If he is not destroyed, this Pepy Neferkare is not destroyed. If he does not mourne, this Pepy Neferkare does not mourn. If he mourns, this Pepy Neferkare will mourn.' Here the king and various deities are mentioned in sentences that stand parallel with each other, with identical sentence structure and word use, substituting only the names of the deities. This creates a situation in which each deities' act of restoration is equally important. Other examples in this text, particularly $\S \S 181-192$, use equivalent sentence structure, but individualise the names of the deities, all of which begin 'In this your name of...'» Para otros elementos literarios y performativos en la composición de los TdP, véanse Reintges 2011, 3-54 y Morales 2016, 23 y ss. 
En esta larga declaración de carácter sacerdotal existe una amplia variedad gramatical que incluye casos donde el beneficiario del texto es a veces identificado como el dios por declaraciones de tipo predicativo ${ }^{27}$, hasta ejemplos en que el nombre wsir «Osiris» a menudo está como una entidad separada del beneficiario del texto ${ }^{28}$.

Frankfort situaba el himno dentro del contexto de las ceremonias que se desarrollaban entre la muerte del rey y la coronación de su sucesor. El peso de la relación padre / hijo = Osiris / Horus en el ritual funerario es de primordial importancia en su análisis, por cuanto todo hijo debía asistir a su padre en ese momento, pero en el caso real el procedimiento se complicaba por la necesidad de reconocer la transfiguración específica que tenía lugar ${ }^{29}$. Este himno, según Frankfort, se recitaría entre el ascenso a la dignidad real y la coronación -mientras el nuevo rey asumía el poder, proclamaba su protocolo, y visitaba los santuarios por todo el país $-^{30}$. En él se presentaba al rey como Osiris a los demás dioses de la Gran Enéada y dioses de la Pequeña Enéada y después el rey-Osiris era invocado como habitante de distintas localidades que asisten así como testigos de esta transfiguración.

A continuación, se presenta la transliteración y traducción de TdP 219, para luego analizar detenidamente la declaración.

\section{$\S 167 \mathrm{a} \underline{d} d d m d w$ tm z3 $p w p n^{31}$ wsir di n.k $s \underline{d b}=f \mathrm{enh}=f$ \\ $\S 167 \mathrm{~b}$ enh $=f$ enh $(w) p n^{32} n m w t=f n i m w t(w) p n$ \\ $\S 167 \mathrm{c} n$ sk=f $n$ sk (w) pn $n$ nhp=f $n n h p$ (w) $p n^{33}$ \\ Para recitar:}

Atum, este de aqui es tu hijo, Osiris, a quien tú has hecho revivir y vive. Él vive y este Unas vive. Él no muere, este Unas no muere. Él no perece y este Unas no perece.

$\S 168 \mathrm{a} \check{s} w z 3=k$ pw pn wsir di $n=k s \underline{d b}=f$ enh $=f$

$\S 168 \mathrm{~b}$ c $n h=f$ enh $(w)$ pn $n$ mwt $=f$ ni mwt $(w)$ pn

$\S 168 \mathrm{c} n$ sk=f $n$ sk (w) pn $n$ nhp=f $n$ nhp (w) pn

Shu, este de aquí es tu hijo, Osiris, a quien tú has hecho revivir y vive. Él vive y este Unas vive. Él no muere, este Unas no muere. Él no perece y este Unas no perece.

$\S 169 \mathrm{a}$ tfnwt $z 3=\underline{t}$ pw pn wsir di $n=\underline{t} s \underline{d b}=f$ enh $=f$

$\S 169 \mathrm{~b}$ enh $=f$ enh (w) pn $n$ mwt=f nimwt (w) pn

$\S 169 \mathrm{c} n$ sk=f $n$ sk (w) pn $n$ nhp=f $n$ nhp (w) pn

27 Hays 2012, 168. «Alongside the statements of identity and the appositival formula Osiris NN, in the Pyramid Texts the name wsir "Osiris" often stands as an entity separate from the text owner», (Hays, 2012, 168).

28 Hays 2012, 168.

29 Si bien la muerte del gobernante (...), exigía que el rey difunto se hubiera convertido en Osiris y que continuara beneficiando al pueblo y apoyando a su hijo y sucesor, Frankfort 1976, 134.

30 Frankfort 1976, 134.

31 Para Griffiths 1980, 228, este $p w$ pn (Osiris sobre ti) es tal vez una referencia al sarcófago; Spiegel 1956, 194, por su parte, cree que hace referencia al b3; «Als Ba lebt der Toter weiter. Das Persona-Pronomen "er" geht hier zugleich auf den Ba und sein Vorbild Osiris, mit welchem der Ba identifiziert ist». Véase Hays 2009b, 197-198.

32 Para Shmakov 2012, 78, este $w$ pn es una «hipóstasis de Osiris, aunque escrita sólo «este Unas».

33 Los parágrafos $\S 168 \mathrm{~d}, \S 169 \mathrm{~d}, \S 170 \mathrm{~d}, \S 171 \mathrm{~d}, \S 172 \mathrm{~d}, \S 173 \mathrm{~d}$ y $\S 174 \mathrm{~d}$ repiten la última parte de los respectivos parágrafos «c», $n h p=f n h p(w)$ pn, y por ello han sido omitidos. Lo mismo para $\S 182 \mathrm{~d}, \S 183 \mathrm{~d}, \S 184 \mathrm{e}, \S 185 \mathrm{~d}$, $\S 187 \mathrm{c}, \S 188 \mathrm{e}, \S 189 \mathrm{e}, \S 190 \mathrm{e}, \S 191 \mathrm{e}, \S 192 \mathrm{e}$. 
Tefnut, este de aqui es tu hijo, Osiris, a quien tú has hecho revivir y vive. Él vive y este Unas vive. Él no muere, este Unas no muere. Él no perece y este Unas no perece.

$\S 170 \mathrm{a} g b \quad z 3=k$ pw pn wsir di $n=\underline{t} s \underline{d} b=f$ enh $=f$

$\S 170 \mathrm{~b}$ enh $=f$ enh $(w)$ pn $n$ mwt=f ni mwt (w) pn

$\S 170 \mathrm{c} n$ sk=f $n$ sk (w) pn $n \operatorname{nhp=f} n \operatorname{nhp}(w) p n$

Geb, este de aquí es tu hijo, Osiris, a quien tú has hecho revivir y vive. Él vive y este Unas vive. Él no muere, este Unas no muere. Él no perece y este Unas no perece.

$\S 171 \mathrm{a} n w t z 3=\underline{t}$ pw pn wsir di $n=\underline{t} n$ s $\underline{d b}=f \mathrm{e} n h=f$

$\S 171 \mathrm{~b}$ enh $=f$ enh $(w)$ pn $n$ mwt $=f$ ni mwt (w) pn

$\S 171 \mathrm{c} n$ sk=f $n$ sk (w) pn $n \operatorname{nhp=f} n \operatorname{nhp}(w) p n$

Geb, este de aquí es tu hijo, Osiris, a quien tú has hecho revivir y vive. Él vive y este Unas vive. Él no muere, este Unas no muere. Él no perece y este Unas no perece.

$\S 172 \mathrm{a} 3 s t$ sn $=\underline{t} p w$ pn wir di $n=\underline{t} s \underline{d b}=f$ enh $=f$

$\S 172 \mathrm{~b}$ enh $=f$ enh (w) pn $n$ mwt=f ni mwt (w) pn

$\S 172 \mathrm{c} n$ sk=f $n$ sk (w) pn $n$ nhp=f $n$ nhp (w) pn

Isis, este tu hermano está aquí, Osiris, a quien tú has hecho revivir y vive. Él vive y este Unas vive. Él no muere, este Unas no muere. Él no perece y este Unas no perece.

$\S 173 \mathrm{a}$ stš $s n=k$ pw pn wsir $d y$ s $\underline{d b}=f \mathrm{enh}=f z z=f$

$\S 173 \mathrm{~b}$ enh $=f \mathrm{e} h \mathrm{~h}=f(\mathrm{w})$ pn ni $m w t=f$ ni $m w t(w)$ pn

$\S 173 \mathrm{c} n$ sk=f $n$ sk (w) pn $n \operatorname{nhp}=f n \operatorname{nhp}(w)$ pn

Seth, este tu hermano está aquí, Osiris, a quien tú has hecho revivir y vive. Él vive y este Unas vive. Él no muere, este Unas no muere. Él no perece y este Unas no perece.

$\S 174 \mathrm{a} n b t$-ḥyt $s n=\underline{t}$ pw pn wsir di=t $s \underline{d} b=f$ enh $=f$

$\S 174 \mathrm{~b}$ enh $=f$ enh (w) pn $n$ mwt =f ni mwt (w) pn

$\S 174 \mathrm{c} n$ sk=f $n$ sk (w) pn $n n \underline{h} p=f n n \underline{h} p$ (w) $p n^{34}$

Neftys, este tu hermano está aquí, Osiris, a quien tú has hecho revivir y vive. Él vive y este Unas vive. Él no muere, este Unas no muere. Él no perece y este Unas no perece.

$\S 175 \mathrm{a} \underline{d h w t i}$ sn=k pw pn wsir $d y$ s $\underline{d} b=f$ enh $=f z z=f$ tw

$\S 175 \mathrm{~b}$ enh $=f$ enh $(w)$ pn $n$ mwt=f ni mwt (w) pn

$\S 175 \mathrm{c} n s k=f n s k(w)$ pn $n \operatorname{nhp}=f n n h p$ (w) pn

Toth, este tu hermano está aquí, Osiris, a quien tú has hecho revivir y vive. Él vive y este Unas vive. Él no muere, este Unas no muere. Él no perece y este Unas no perece.

34 Tanto $\$ 174 \mathrm{c}-175$ a como luego $\$ 184 c$-d son dos de los cuatro casos de inserción de una frase en la cámara funeraria de la pirámide de Unas, Mathieu 1996, 303-304. 
$\S 176 \mathrm{a} h r$ it $=k$ pw nn wsir di $n=k s \underline{d b}=f$ enh $=f$

$\S 176 \mathrm{~b}$ enh=f enh (w) pn n mwt=f ni mwt (w) pn

$\S 176 \mathrm{c} n s k=f n s k(w)$ pn $n \operatorname{nhp}=f n n h p$ (w) pn

Horus, este de aqui es tu padre, Osiris ${ }^{35}$, a quien tú has hecho revivir y vive. Él vive y este Unas vive. Él no muere, este Unas no muere. Él no perece y este Unas no perece.

\$177a ps $\underline{d t}$ c $3 t$ wsir $p w$ pn di $n=\underline{t} n s \underline{d b}=f$ e $n h=f$

$\S 177 \mathrm{~b}$ enh $=f$ enh $(w)$ pn $n$ mwt=f ni mwt (w) pn

$\S 177 \mathrm{c} n s k=f n s k(w)$ pn $n \operatorname{nhp}=f n n h p$ (w) pn

Gran Eneada ${ }^{36}$, este Osiris está aquí, a quien tú has hecho revivir y vive. Él vive y este Unas vive. Él no muere, este Unas no muere. Él no perece y este Unas no perece.

$\S 178 \mathrm{a} p s \underline{d t} n \underline{d s t}$ wsir $p w$ pn di $n=\underline{t} n$ s $\underline{d b}=f$ enh $=f$

$\S 178 \mathrm{~b} \times n h=f$ enh (w) pn $n$ mwt $=f$ ni mwt (w) pn

$\S 178 \mathrm{c} n$ sk=f $n$ sk (w) pn $n$ nhp=f $n$ nhp (w) pn

Pequeña Eneada ${ }^{37}$, este Osiris está aquí, este tu hijo está aquí, Osiris, a quien tú has hecho revivir y vive. Él vive y este Unas vive. Él no muere, este Unas no muere.

$\S 179 \mathrm{a}$ niwt $z 3=\underline{t}$ pw pn wsir $\underline{d} d \mathrm{n}=\underline{\mathrm{t}}$ irf $m s t \mathrm{n} i \mathrm{t}=\underline{\mathrm{t}} \mathrm{n}$

$\S 179 \mathrm{~b}$ sk $n=\underline{t} n=f r=f$ wp $r 3=f$ in $z 3=f$ hr $m r y=f$

$\S 179 \mathrm{c}$ tnw $\mathrm{c} w \mathrm{t}=\mathrm{f}$ in $n$ ntrw

niwt ${ }^{38}$, este tu hijo Osiris está aquí, de quien tú has dicho: "Alguien ha nacido de mì», tú dices, y limpias su boca, su boca ha sido abierta por su hijo Horus, quien es su amado, sus miembros han sido considerados por los dioses.

$\S 180 \mathrm{a} \times n h=f$ enh (w) pn $n$ mwt=f ni mwt (w) pn

$\S 180 \mathrm{~b} n$ sk $=f n$ sk (w) pn $n$ nhp=f $n$ nhp (w) pn

$\S 180 \mathrm{c} n h p=f n h p(w) p n$

Él vive y este Unas vive. Él no muere, este Unas no muere. Él no perece y este Unas no perece. Él no es llevado y este Unas no será llevado.

$\S 181$ a $m r n=k i m(i) i w n w n \underline{d} d d n \underline{d} d=f m \underline{d} d t=f$ $\S 181 \mathrm{~b}$ c $n h=f$ enh (w) pn $n$ mwt=f ni mwt (w) pn 181c $n$ sk=f $n$ sk (w) pn $n \operatorname{nhp=f} n \operatorname{nhp}(w)$ pn $n h p=f n h p$ (w) pn

En tu nombre de El-de-Heliopolis-mientras-permanece-en-su-necrópolis, Él vive y este Unas vive. Él no muere, este Unas no muere. Él no perece y este Unas no perece. Él no es llevado y este Unas no será llevado.

35 Th. Allen 1916, 22, llama a esta relación como «Genealógica».

36 Atum, Shu, Tefnut, Nut, Geb, Isis, Osiris, Neftis y Seth.

37 Toth, Maat, Anubis, Khnum y Horus.

38 Para Faulkner $(1969,47)$ la traducción es «City», «...either the tomb of Osiris or $n n t$ «the lower sky, but the absence of the determinative

que «Nunet» es para Piankoff $(1968,66)$ la traducción apropiada de $\curvearrowright$. Para Sethe 1929, 87, «“Ciudad” es la tumba de Osiris o nnt como cielo inferior. ...und war wird entweder der Göttin des unterirdischen Himmels, die "Naunet" gemeint sein, die freilich sonst wohl stets mit Heimat des Totes "Die Stadt"». El uso del determinativo de ciudad (niwt) o el hecho de que a Nut se la considere en ocasiones una diosa embarazada de los dioses podría estar relacionado con esta línea de los TdP. 
$\S 182 \mathrm{a} m r n=k i m(i)$ end $\underline{\mathrm{d}} \mathrm{h} \mathrm{r}$ tp $\mathrm{s} p 3 w t=\beta^{39}$

$\S 182 \mathrm{~b}$ c $n h=f$ enh $(w)$ pn $n$ mwt $=f$ ni mwt $(w)$ pn

$\S 182 \mathrm{c} n$ sk=f $n$ sk (w) pn $n$ nhp=f $n$ nhp (w) pn

En tu nombre de El-de-Andjety, jefe de sus nomos, Él vive y este Unas vive. Él no muere, este Unas no muere. Él no perece y este Unas no perece.

$\S 183 \mathrm{a} m \mathrm{rn}=\mathrm{k} i \mathrm{~m}$ (i) srkt $\mathrm{k} 3 \mathrm{htp}$

$\S 183 \mathrm{~b}$ enh $=f$ enh (w) pn $n$ mwt=f ni mwt (w) pn

$\S 183 \mathrm{c} n$ sk=f $n$ sk (w) pn $n$ nhp=f $n$ nhp (w) pn

En tu nombre de El-de-la-Mansión-de-Selkis, el k3 descansa. Él vive y este Unas vive. Él no muere, este Unas no muere. Él no perece y este Unas no perece.

$\S 184 \mathrm{a} m \mathrm{rn}=\mathrm{k} i m(i) z h-n \underline{t} r^{40} i m(i) k 3 p$

$\S 184 \mathrm{~b} d b n(=i)$ tzti inikti

$\S 184 \mathrm{c}$ enh=f enh (w) pn n mwt=f ni mwt (w) pn

$\S 184 \mathrm{~d} n$ sk=f $n$ sk (w) pn $n$ nhp=f $n$ nhp (w) pn

En tu nombre de El-de-la-tienda-del-dios, Que-está-en-la-en-fumigación, Elque-está-en-el-cofre, El-que-está-en-el-Arca, El-que-está-en-el-Saco ${ }^{41}$, Él vive y este Unas vive. Él no muere, este Unas no muere. Él no perece y este Unas no perece.

$\S 185 \mathrm{a} m \mathrm{rn}=\mathrm{k} i \mathrm{~m}(i) \underline{h t} \mathrm{p}$ 3er

$\S 185 \mathrm{~b}$ enh $=f$ enh (w) pn $n$ mwt=f ni mwt (w) pn

$\S 185 \mathrm{c} n$ sk=f $n$ sk (w) pn $n$ nhp=f $n$ nhp (w) pn

En tu nombre de El-que-está-en-la-Capilla-Blanca-de-madera-p3er, Él vive y este Unas vive. Él no muere, este Unas no muere. Él no perece y este Unas no perece.

§186a $m r n=k$ imi $s 3 h t r=k r p t$ tr $=k r t 3$

$\S 186 \mathrm{~b}$ wsir pšr $h r=k m 3=k n$ (w) pn

$\S 186 \mathrm{c} m t w t=k$ prt $i m=k$ spdt $t^{42}$

En tu nombre de El-de-Orion, tu tiempo está en el cielo, tu tiempo está en la tierra.

Osiris, vuelve tu rostro hacia este Unas, tu simiente que surgió de ti es eficaz.

$\S 187 \mathrm{a} \bullet \mathrm{nh}=f$ enh $(w)$ pn $n$ mwt=f ni mwt (w) pn

$\S 187 \mathrm{~b} n$ sk=f $n$ sk (w) pn $n$ nhp=f $n$ nhp (w) pn

Él vive y este Unas vive. Él no muere, este Unas no muere. Él no perece y este Unas no perece.

39 Mathieu 2010, 90, cita 77

40 De acuerdo a Hoffmeier 1981, 167-177, «the $z h$-n $n \underline{t} r$ (and the $i b w$ ) were purifications tents whose origins could be traced to a theological model and functioned in conjunction with the re-enactment of Re's purification in a setting that best emulated the mythic archetype»; ver también Brovarski 1977, 107-115.

41 Según Griffiths 1980, 21, «the god's tent is the mummification tent, the 'fumigation' refers to the incense-burning which a part of several rites, the 'box' points to sarcophagus, the 'shrine' to the funerary shrine carried in a boat, and the 'cloth' to the bindings around the mummy».

42 Para Griffiths 1980, 20-21, spdt «is an error for $s p d$, and that the reference is to (Horus) Sopd, who is said in $632 \mathrm{~d}$ to have resulted from the union of Osiris-King (as Orion) and Isis (as Sothis); in the present context (...) the King is identified with the offspring of Osiris, so that a form of Horus is appropriately involved». Sin embargo, la presencia de la marca del femenino $=t$ conspiraría contra esta afirmación por cuanto la palabra $s p d t$ sería un sustantivo femenino y no un adjetivo. 
$\S 188 \mathrm{a} m \mathrm{rn}=\mathrm{kim}(\hat{\imath}) \mathrm{dp}$

$\S 188 \mathrm{~b} c=k$ swt iht $z 3 t=k$ h $3 \mathrm{tw} i \mathrm{~m}=s$

$\S 188 \mathrm{c}$ enh=f $\mathrm{enh}(\mathrm{w})$ pn $n$ mwt=f ni mwt (w) pn

$\S 188 \mathrm{~d} n s k=f n s k(w)$ pn $n \operatorname{nhp}=f n n h p(w)$ pn

En tu nombre de El-de-Dep, que tu mano esté cerca de tu comida, tu hija, provéete con ella. Él vive y este Unas vive. Él no muere, este Unas no muere. Él no perece y este Unas no perece.

$\S 189 \mathrm{a} m \mathrm{rn}=\mathrm{k} i m(i)$ hwt wr $k 3$

$\S 189 \mathrm{~b} c=k$ swt iht $z 3 t=k$ h 3 tw $i m=s$

$\S 189 \mathrm{c}$ enh $=f$ c $n h$ (w) pn $n$ mwt=f ni mwt (w) pn

$\S 189 \mathrm{~d} n$ sk=f $n$ sk (w) pn $n$ nhp=f $n$ nhp (w) pn

En tu nombre de El-que-está-en-el-palacio-del-gran-Toro, que tu mano esté cerca de tu comida, tu hija, provéete con ella. Él vive y este Unas vive. Él no muere, este Unas no muere. Él no perece y este Unas no perece.

$\S 190 \mathrm{a} m \mathrm{rn}=\mathrm{kim}(i)$ wnw $r s w$

$\S 190 \mathrm{~b} c=k$ swt $i h t$ z $3 t=k$ htm $\underline{t} w i m=s$

$\S 190 \mathrm{c}$ enh=f enh (w) pn n mwt=f ni mwt (w) pn

$\S 190 \mathrm{~d} n$ sk=f $n$ sk (w) pn $n$ nhp=f $n$ nhp (w) pn

En tu nombre El-que-está-en-Unu-del-Sur, que tu mano esté cerca de tu comida, tu hija, provéete con ella. Él vive y este Unas vive. Él no muere, este Unas no muere. Él no perece y este Unas no perece.

$\S 191 \mathrm{a} m \mathrm{rn}=\mathrm{kim}(i)$ wnw $m$ ht

$\S 191 \mathrm{~b} c=k$ h 3 iht $z 3 t=k$ htm $\underline{t}$ w $i m=s$

$\S 191 \mathrm{c}$ enh=f enh (w) pn n mwt=f ni mwt (w) pn

$\S 191 \mathrm{~d} n$ sk=f $n$ sk (w) pn $n$ nhp=f $n$ nhp (w) pn

En tu nombre de El-que-está-en-Unu-del-Norte, que tu mano esté cerca de tu comida, tu hija, provéete con ella. Él vive y este Unas vive. Él no muere, este Unas no muere. Él no perece y este Unas no perece.

§192a $m r n=k i m(i)$ niwt šw

$\S 192 \mathrm{~b}$ wnmt $n=k$ irt=i. šn ht $=k$ hr $r=s$ ifh $n=k s \quad z 3=k$ hr $r \quad n h=k i m=s^{43}$

$\S 192 \mathrm{c} e n h=f \mathrm{enh}(\mathrm{w})$ pn ni $m w t=f$ ni $m w t(w)$ pn

$\S 192 \mathrm{~d} n$ sk=f $n$ sk (w) pn $n$ nhp=f $n$ nhp (w) pn

En tu nombre de El-que-está-en-la-Ciudad-de-los-Lagos, lo que tú has comido es un ojo, y tu estómago se ha hinchado a causa de él. Tu hijo Horus te lo ha cedido para que puedas vivir con él. Él vive y éste Unas vive. Él no muere, este Unas no muere. Él no perece y este Unas no perece.

$\S 193 \mathrm{a} d t=k \underline{d} t \mathrm{nt}(w)$ pn iwf=kiwf $n$ (w) pn

$\S 193 \mathrm{~b} k s w=k \mathrm{ksw}(w)$ pn

$\S 193 \mathrm{c} s b=k s b(w)$ pn $s b(w)$ pn $s b=k^{44}$

43 Para una interpretación de esta frase con énfasis en el ojo de Horus, ver Edwards 1995, 63-64 y 182.

44 Para referencias a este parágrafo, ver Martin 2013, 176-177. 
Tu cuerpo es el cuerpo de Unas, tu carne es la carne de Unas, tus huesos son los huesos de Unas. Tu vas, este Unas va, tu vas ${ }^{45}$.

La complejidad y riqueza del texto es ostensible. La variedad temática descubre una multiplicidad de situaciones que conforma la secuencia litúrgica en la que opera TdP 219. Es por ello que desde la sinopsis de lo que conlleva, esto es, la identificación del faraón con Osiris, se detalla una serie de cuestiones que le dan sustento y que a continuación intentaremos analizar. Una descripción de esta extensa declaración es dada por Mathieu;

Le TP 219 (...), présente au même emplacement dans toutes les pyramides à textes, constitue un véritable exposé dogmatique de la Réforme osirienne, dans ses dimensions théologique et géographique. Y sont détaillées en effet, dans une première partie (stances 1-12), la généalogie héliopolitaine d'Osiris (Atoum, Chou, Tefnout, Geb, Nout, Isis, Seth, Nephthys, Thot, Horus, Grande Ennéade, Petite Ennéade), et, dans une seconde partie (stances 14-24), la diffusion géographique du culte osirien, à partir d'Héliopolis. Cette seconde partie est elle-même divisée en deux groupes de cinq stances: le premier groupe (14-18) évoque Héliopolis, Busiris, les sanctuaires de Kahotep, d'Anubis et de Hedjourou, tandis que le second (20-24) évoque Bouto, Memphis, Hermopolis du Sud, Hermopolis du Nord et le sanctuaire de «Celui qui est dans la Cité des nomes». Les deux groupes sont séparés par une stance «astronomique» (19), dévolue à la constellation Sah (notre Bouclier d'Orion). L'absence remarquable d'Abydos, dans ce panorama territorial, pose une vraie question; la réponse, sous toute réserve, pourrait tenir à la date de rédaction de la formule ${ }^{46}$.

Como primera herramienta de aproximación, y a los efectos de comprender un texto tan extenso, una subdivisión temática es necesaria. Para ello, un gráfico resulta más esquemático que una larga prosa;

$\begin{array}{ll}\S 167 \text { a-179c ...................... Presentación del difunto como Osiris a las divinidades } \\ \text { Afiliación: Hijo } & \S 167 \text { hijo de Atum } \\ & \S 168 \text { hijo de Shu } \\ & \S 169 \text { hijo de Tefnut } \\ & \S 170 \text { hijo de Geb } \\ & \S 171 \text { hijo de Nut } \\ & \S 179 \text { hijo de Nunet } \\ & \S 172 \text { hermano de Isis } \\ & \S 173 \text { hermano de Seth (vida y castigo) } \\ & \S 174 \text { hermano de Neftys }\end{array}$

45 Respecto al tema de la relación entre un dios (o un difunto Osiris) y las partes del cuerpo de los dioses, véanse Beinlich 1984 y la reseña del mismo trabajo, Pantalacci 1987, 108-123.

46 Mathieu 2010,91. La posición del texto - en líneas generales-como consecuencia de su función y ordenación de los corpora de TdP en las pirámides de los reyes del Reino Antiguo, también aparece en el Reino Medio como parte de la selección de textos transmitidos en el tiempo y codificados junto a los Textos de los Sarcófagos, lo que expresa un nuevo posicionamiento en las colecciones. En segundo lugar, y no menos importante, es el hecho de que TdP 219 aparece también en las colecciones para las reinas del Reino Antiguo -Ibi, Neith e Iput- lo que nos plantea la cuestión acerca de un uso distintivo pero similar en significado a los reyes. 
Afiliación: Padre

Relación: Conservación

Relación: Ontológica

Relación: Geográfica

y de Continuidad

Locación: Bajo Egipto

Locación: No-especificada

Relación: Ontológica

Relación: Ontológica
$\S 175$ hermano de Toth (vida y castigo)

$\$ 176$ padre de Horus

$\S 177$ La Gran Enéada le (a Osiris) ha hecho revivir y vivir

$\$ 178$ La Gran Enéada le ha hecho vivir

$\S 180 a-c$ Confirmación de la relación Osiris / Faraón

$\S 181 \mathrm{a}-192 \mathrm{e}$ Osiris en diferentes localizaciones

$\S 181$ On

$\S 182$ Andjety

$\S 183$ Habitante en-la-Mansion-de-Selkis ${ }^{47}$

$\S 188$ Dep

\$189 Palacio-del-Gran-Toro

$\S 190$ Unu del Sur

$\S 191$ Unu del Norte

$\S 184$ Habitante de-la-tienda-del-dios

$\$ 185$ Capilla-Blanca de-madera- $p 3 e r$

\$186 Habitante-de-Orión

relación Osiris/Faraón §187a-c Identificación Osiris/Faraón

relación Osiris/Faraón §193a-c Identificación Osiris/Faraón

En la p. 5 marcamos el peso que en este ritual tiene la relación padre hijo como espejo de la fórmula Horus sucediendo a Osiris. De este modo, un claro trasfondo de sucesión genealógica explica parte de los textos funerarios reales y en este texto ritual en particular hay alusiones explícitas. En la fórmula §176a $h r$ it $=k p w n n$ wsir «Oh Horus, éste ${ }^{48}$ de aquí es tu padre Osiris» queda expresada esta relación de trascendental importancia para que el rey difunto -Osiris- continuara beneficiando al pueblo y apoyando a su hijo y sucesor con todo el poder sobrehumano del que disponía ${ }^{49}$.

Con la presentación del difunto a las divinidades se generaba la asimilación del faraón difunto como Osiris a la corporación divina. En esta exposición que en realidad concluye en incorporación, el faraón resume su doble condición divina, por su investidura y por su condición de muerto.

A excepción de los parágrafos vinculados a Seth y a Toth (en los que además de dar vida, también castigan), al faraón / Osiris se le restablece, se le hace vivir, no se le destruye ni muere ni se lamenta ni llora, en una estructura formularia que se reitera para cada una de las divinidades y para la Gran y Pequeña Enéada.

La misma estructura se mantiene a continuación en las referencias a diferentes espacios y localidades, la mayoría vinculada al Bajo Egipto, lo que ha llevado a suponer que TdP 219 conserva, al menos en parte, el texto prototípico de un enterramiento del Bajo Egipto. La adscripción geográfica se debe a que las ciudades citadas en la letanía final se identifican con lugares del Delta occidental ${ }^{50}$.

47 Ver von Känel 1983, cols. 830-833.

48 Los pronombres demostrativos «éste», «ése», «aquél», son interesantes por cuanto expresan proximidad o lejanía; «L'emploi du déictique de proximité $p n / t n / n n$ suposse la présence matérielle du signifié dans un context spatial inmédiat, en fonction du centre déictique ("deictic center") que constitue l'espace funéraire et le défunt en particulier», Mathieu 2016, 409.

49 Frankfort 1976, 134

50 Molinero Polo 1998, 267. Del mismo modo, Sethe 1929, 92; Griffiths1980, 21. 
Por último, el texto se inicia con una presentación del rey difunto a la Enéada, pero los testimonios más antiguos de este colegio divino son muy posteriores a la hipotética fecha predinástica del reino septentrional, lo que aboga también por una datación posterior ${ }^{51}$.

No obstante, además de estas localizaciones del Bajo Egipto, la liturgia completa tiene dos declaraciones, TdP 220-221, en las que el Rey era coronado con la corona roja del Bajo Egipto $^{52}$.

Y si bien es cierto que «the spell is fully of inconsistencies, however, in its grammatical structure in relation to the gods, for in the whole of the first part Osiris is alluded to in the third person $»^{53}$, ésta última división es ilustrativa de un corte en el conjunto textual.

La primera parte -escrita en tercera persona- comienza en Pir. $§ 167$ a y finaliza en $\$ 180$ c y coincide con la presentación del faraón / Osiris al conjunto de los dioses. En el sufijo $=k$ el rey difunto es introducido si no en una posición subalterna, sí en una «aspiracional». El sacerdote instaura, presentándolo, al rey como miembro de la familia divina. Inmediatamente, la segunda persona es reemplazada por la tercera $=f$ una vez que la introducción a la corporación divina haya concluido en la recepción de su par, el faraón / Osiris. La mayor formalidad que supone la tercera persona en la segunda parte de cada uno de los parágrafos se vincula justamente con la incorporación, mientras que la informalidad que puede conllevar el sufijo $=k$, tiene una mayor relación con un contexto de presentación.

El sacerdote delegado para esta extensa declaración cumple la doble tarea de solicitar la aceptación, al presentar al rey entre los dioses, y de expresar el deseo de que todo aquello que suceda a los dioses sea extensivo al faraón. La relación sería entonces:

\author{
Sufijo \\ Primeras Partes \\ Segunda Persona \\ $=k(\mathrm{Du}-$ Texte $)$ \\ Presentación del difunto \\ (petición de aceptación)
}

\author{
Sufijo \\ Segundas Partes \\ Tercera Persona \\ $=f($ Er-Texte $)$ \\ Asimilación Rey / Osiris \\ (recepción e inclusión)
}

Finalmente, en Pir. §193a-c que culmina la declaración, se continúa con la existencia del faraón / Osiris, haciéndolos coincidir hasta la identificación ${ }^{54}$; cuerpo, carne y huesos conforman una unidad ontológica entre el dios y el rey, del mismo modo que los desplazamientos se confunden -identificándose-.

\footnotetext{
51 Molinero Polo 1988, 267.

52 «These two utterances (...) are closely connected, being part of the ritual accorded to the crown of Lower Egypt», Faulkner 1969, 48. De acuerdo a Sethe 1929, 100, esta es «ein Spruch, der vom Priester gesprochen wurde, wenn der lebende König die Personifikation der unterägyptische Königskrone, die mit der Götting Buto der Schutgöttin des unterägyptischen Königstum identisch war”. En TdP 555 (\$1373a- \$1378c) el Rey es coronado con la corona blanca -vinculada al Alto Egipto- y la corona verde -vinculada al Bajo Egipto- pero sin ningún tipo de ritual. «Nekhbet was identified with the White Crown of Upper Egypt and Wadjyt with the Red (sometimes green) Crown of Lower Egypt», Pinch 2002, 211. Para un estudio integral de las coronas egipcias, ver Goebs 2008.

53 Griffiths 1980, 137.

54 Según Smith 2006, 335, «If I asked to select the Egyptian verb that best characterizes the relationship between the Osiris or Hathor of a person and those deities, then (...) I would opt for "become", but rather "follow", "serve". The Osiris of a deceased person does not become Osiris, but rather a member of his retinue». En el
} 
Estas referencias aluden al proceso de momificación y su consiguiente posibilidad de pervivencia eterna. Osiris fue el primer momificado evitando de este modo la putrefacción y la definitiva disgregación ${ }^{55}$; el rey, al asumir este derrotero de Osiris, procura el mismo destino a instancias de su hijo y sucesor Horus.
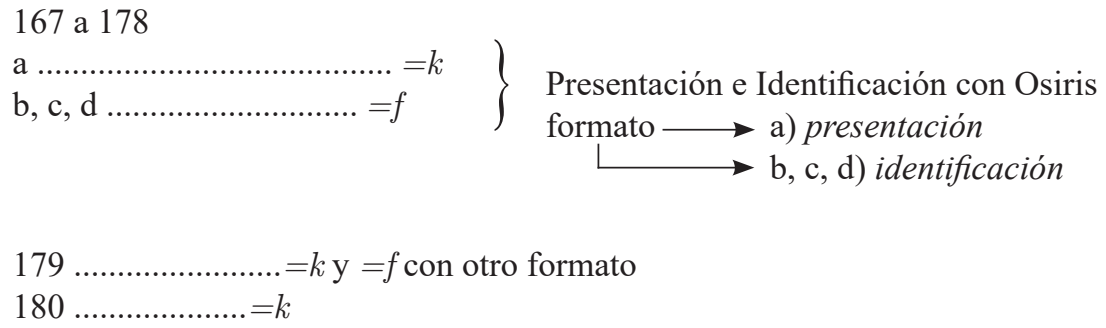

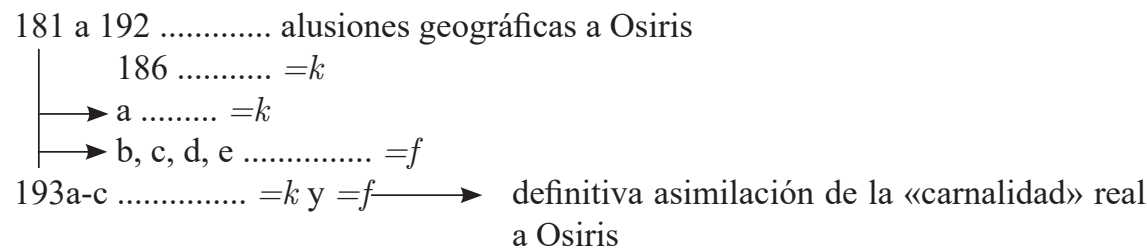

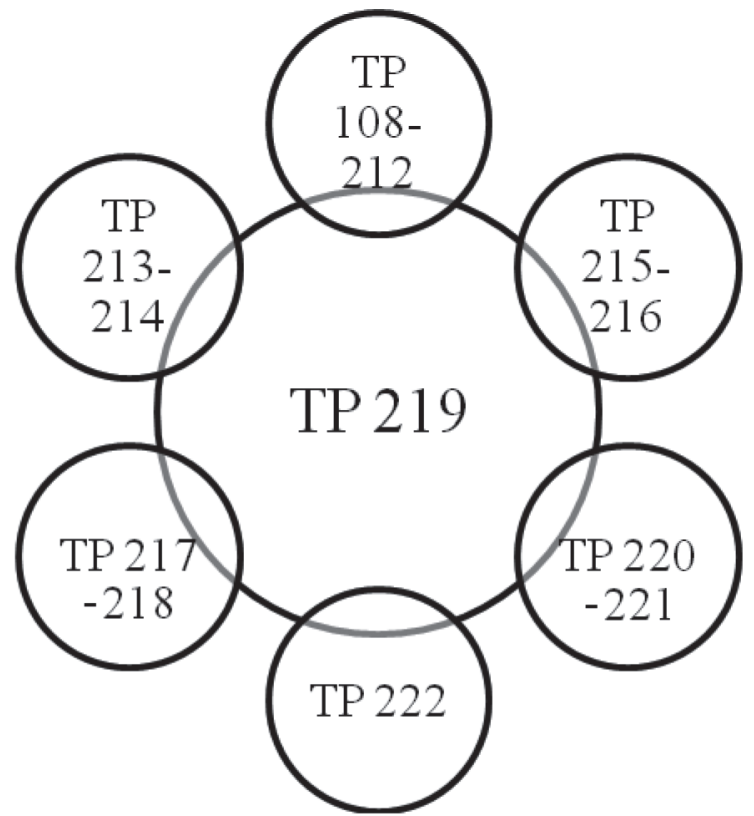

Figura 1. Centralidad y relación de TP 219 en la secuencia litúrgica TP 213-222 con sus antecedentes preliminares

mismo sentido, «the locution wsir NN, in which the name of the deceased is preceded by that of Osiris, does not indicate identity between them. The use of this locution was restricted to certain contexts; it served initially to identify the dead as recipients of sustenance under the auspices of Osiris, although it soon came to be a more generic marker of the deceased's association with the god», Smith 2013, 89-90.

55 Esta disgregación reviste un carácter físico y uno social; véase Assmann 2005, sobre todo Capítulo 2. 
Las segunda y tercera personas confirman la pertenencia del texto a una liturgia sacerdotal en beneficio del rey. Éste, como testigo silente, se identificaba con Osiris y se asimilaba a su ciclo que evitaba la desagregación corporal y se perpetuaba indefinidamente. La secuencia completa de la liturgia se estructura de modo que TdP 219 opera como nexo entre la propedéutica que supone la provisión de alimentos y perfumes $^{56}$ y la consecuente asunción del rey al cielo (TdP 213-214), o como una estrella (TdP 215-216), o uniéndose al dios-sol (TdP 217) o asumiendo la autoridad en el Más Allá (TdP 218), y la posterior coronación real asumiendo el rey como Osiris y en pleno ejercicio de su investidura (TdP 222) -unión del rey con el dios sol- pero, a diferencia de TdP 217, el rey habla con el sacerdote delegado en primera persona. En el medio, TdP 219 se erige en vínculo ontológico necesario, por la asunción del rey definitivamente como Osiris y desde allí su búsqueda y ascenso y descenso con $\mathrm{Re}^{57}$.

\section{TdP 219 como antecedente de los Libros del Más Allá del Reino Nuevo}

Es necesario introducir la compleja problemática de la intertextualidad en lo que concierne a la tríada Faraón-Re-Osiris entre los Textos de las Pirámides y los Libros del Más Allá del Reino Nuevo ${ }^{58}$, considerando que «Osiris et Rê sont compris comme formant les composantes complémentaires d'une seule et même entité à dimension cosmique» ${ }^{59}$.

En la relación entre Re y Osiris en los Libros del Más Allá del Reino Nuevo, la inclusión dentro del esquema general de metamorfosis solar de la capacidad regenerativa del último plantea el «espíritu» de los textos, esto es, Osiris devenido en un aspecto o carácter del dios solar. No hay que olvidar su función primigenia de dios de la fertilidad y su preeminencia sobre el mundo de los muertos, hasta su posterior identificación en y con Re, que es quizás la mayor originalidad de los textos. Esto si pensamos que en los TdP su subordinación era proporcional a la primacía solar, y los canales de salvación y transfiguración se presentaban complementarios cuando no divergentes ${ }^{60}$.

Quien más claramente expresó este continuum fue Allen:

In the Pyramid Texts this goals ( $p r t m$ hrw «to emerge in the daytime») at the end of the king`s process of resurrection from the sarcophagus, as it does for the sun's nightly passage through the Duat. The Pyramid Texts combine in one corpus the same vision of rebirth that the New Kingdom divided into the Book of the Dead on the one hand and the «Netherworld» books on the other. While the texts them-

56 Desde TdP 108 hasta TdP 212, sin contar declaraciones anteriores de presentación de sandalias -TdP 106- o incluso anteriores referidas a la presentación de ungüentos-TdP 72, TdP 73, TdP 74, TdP 75, TdP 76, TdP 77 y TdP 78-.

57 «Ra and Osiris are depicted as a real alter ego of the king representing the actual core of his religious-eschatological system while the stars only occasionally take part in it. The statistic account of their mentions in the pyramid texts significantly confirms this difference», Nuzzolo 2010, 186, cita 44.

58 Se incluyen bajo esta denominación al Libro del Amduat, el Libro de las Puertas, el Libro de las Cavernas y el Libro de la Tierra.

59 Mauric-Barbiero 2010, 188.

60 Cfr. Barta 1981, 149-150, para quien no hay enfrentamiento o contraposición funcional entre un destino osiriano y uno solar, sino complementariedad. 
selves are ancestral to the Book of the Dead, the architecture they decorate is conceptually identical to the cosmic geography of the sun's nightly journey described in the Amduat and similar New Kingdom creations. In this respect, the pyramid substructure can be read as a concrete expression of the Amduat's title: $z h 3 w n$ et imnt «Description of the Hidden Space ${ }^{61}$.

En los Libros del Más Allá del Reino Nuevo ambos dioses son parte de un mismo proceso que los involucra y complementa, constituyen fases de un movimiento cíclico que los unifica y sostiene la estructura universal. El dios solar difunto, que ha descendido al Mundo Inferior, se convierte en un «Osiris», toma el papel, la naturaleza y la figura de este dios, que permanece ejemplar para el destino mortal ${ }^{62}$. Osiris y Re son el punto clave en la regeneración de los poderes del sol y los límites, antes jerárquicamente establecidos, parecen difuminarse en las representaciones, los textos y los respectivos procesos involucrados.

The idea that the sun god Re becomes a $b a$ offered New Kingdom theologians a new solution to a long-standing problem: how to explain adequately the relation between Re, who as the nocturnal sun spends time in the underworld, and Osiris, the ruler of this same underworld. According to the newfound explanation, Re himself becomes the $b a$ of Osiris. Uniting with the body of the underworld god each night, he penetrates him with his light and thereby awakens new life (...). Thus Osiris becomes incorporated into the daily course of the sun ${ }^{63}$.

La relación entre ambos dioses que puede parecer o difusa o ambigua en los TdP, alcanza un grado de contigüidad ontológica en los Libros del Más Allá del Reino Nuevo que explica y descubre aquel estadio previo de los TdP. Ello no se vincula a un sesgo evolutivo en las ideas religiosas, sino con el hecho de que «the union of Osiris and Re is a connecting thread in Egyptian religion which stretches from the $5^{\text {th }}$ Dynasty until Roman times. It is a topic which has received some exemplars, but all too brief, analyses $\rangle^{64}$.

Si bien algunos autores afirman que la noción de una unión entre el dios solar y el dios de los muertos está atestiguada desde los Textos de los Sarcófagos en adelante ${ }^{65}$, un antecedente - si bien indirecto- nos presenta TdP 219 y la secuencia y sub-secuencia ${ }^{66}$ que la contiene. Para ello debemos seguir la estructura de la liturgia para dimensionar lo que pretende sostenerse. Si se tiene en cuenta que TdP 217 Pir $\S 160 a-c$ en particular, marca la relación filial del faraón con Re (Atum-Re), y con ello la unión entre el faraón y el dios-sol, y que en la posterior PT 218 Pir §163a-d y Pir $§ 164 a$ el faraón es presentado como un $i h m$-sk («espíritu imperecedero») ante

61 Allen 1994, 28.

62 Hornung 1999, 145.

63 Hornung 1992, 109-110.

64 DuQuesne 2006, 32.

65 Jørgensen 2011, 74. En el mismo sentido, Rößler-Köhler 1980, col. 258, afirma que «eine systematische Verbindung beider Vorstellunkomplexe (heavenly - Re- and earthly -Osiris-) ist in der Pyr. nicht gegeben, der Aufenthalt des kgl. Toten im unterirdischen Jenseits wird z.B. nicht notwendige Voraussetzung zum Aufstieg in den Himmel oder als Sonnenlauf angeschlossene zyklische Wiederkehr geschildert, sondern stellt sich als relativ isoliertes Intermezzo dar".

66 Hays 2012, 475. Me refiero en este caso a TdP 218, Sub-secuencia 86, Dependiente de la Secuencia 37. 
Osiris, puede extraerse ciertos elementos que podrían hacernos suponer una vinculación entre la tríada faraón/Re/Osiris.

Veamos ambos textos en secuencia continua para luego ponerlos en contexto y relación:

$\S 160 \mathrm{a}$ tm re ii $n=k z 3=k$ ii $n=k(w)$

$\S 160 \mathrm{~b}$ sic $n=k$ sw sn $n=k$ sw $m$ hnw $n(w i)=k$

$\S 160 \mathrm{c} z 3=k$ pw $n \underline{d} t=k n \underline{d} t$

Atum-Re, tu hijo viene a ti, Unas viene a ti. Que él ascienda a ti. Abrázalo en tu abrazo. Este es el hijo de tu cuerpo eternamente.

§163a ii $r=f(w)$ pn hwrr ${ }^{67}$ 3h ihm-sk

$\S 163 \mathrm{~b}$ sn $i r=k$ sn $n$ n $i r=t$ nn $i r=t$ wr $i r=k$ w $\underline{d}$ i $r=k$

$\S 163 \mathrm{c} n \mathrm{hmhm}$ ir $=k$ $n$ tr $=k$ igr im

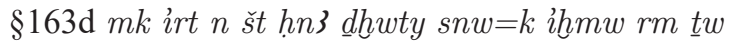

$\S 164$ a ist hn\} nbt-hyt ink ir $=\underline{t} n$ ink ir $=\underline{t} n$

Este Unas ha venido, cansado de los Nueve Arcos, un 3 h imperecedero, que te supera y lo superas, más grande y más fuerte que tú, que grita más fuerte que tú y son más aclamados que tú, y no está alli tu tiempo. Esto es lo que Seth y Toth han hecho, tus hermanos que no lloran por ti. Oh Isis y Neftys vengan juntas, vengan juntas, únanse, únanse.

§160a-c 1. El faraón llega a su padre Re-Atum

1.1 Unas va hacia Re-Atum

1.1.1 Re-Atum lo hace ascender

1.1.2 Re-Atum lo abraza

1.2 El faraón surge eternamente el cuerpo de Re-Atum

$\S 163 \mathrm{a}-\mathrm{d}$ y $\S 164 \mathrm{a} \quad 2$. Unas, como un 3 h imperecedero, llega a Osiris.

2.1. Unas supera a Osiris

2.1. Unas está cansado, más grande y más aclamado que Osiris

2.2 No hay más tiempo para el rey ${ }^{68}$

2.3 Acciones de Seth y Toth

2.4. Isis y Neftys se reúnen, se reúnen juntas, juntas...

2.4.1 Acciones de Isis y Neftys

La relación filial entre el faraón y Re-Atum al que el primero alcanza en un movimiento ascendente, antecede a su enlace con Osiris. La asunción real del estado de 3 h previamente a la llegada ante Osiris, confirma esta aseveración. En este sentido, si bien no se ratifica en forma explícita una relación sistemática entre ambos complejos (solar y osiriano), sí puede concebirse un vínculo de carácter ontológico tan ostensible en los Libros del Más Allá del Reino Nuevo ${ }^{69}$.

$67 \quad$ Wb III 248.

68 Faulkner 1969, 46 traduce «Has stilled», y entonces «The king takes over the role of Osiris as king of the dead»; de allí que haga una analogía con la asunción del rey del papel de Osiris como rey de de los difuntos.

69 Una frase que expresa cabalmente esta situación aparece también en Pir. §164d, imntyw imw t3 $n$ (w) pn «los Occidentales que están en la tierra pertenecen a este Unas». 
El tono inflexivo que tal unión conlleva establece las esferas de influencia, posibilidades y potencialidades de ambos dioses así como sus respectivas conclusiones -la permanencia de la carne en las regiones osirianas y la salida de Re que aquí comenzó su transformación irreversible ${ }^{70}$.

Re and Osiris are not identical, but they unite every night in the depth of the earth to form one entity, of which Re is the Ba-Soul and Osiris the body ${ }^{71}$.

De esta unión -contenida en una liturgia en los TdP y grabada en las tumbas reales en los Libros del Más Allá del Reino Nuevo-, se desprende la conclusión de una pareja de dioses en pleno intercambio de capacidades con fines genésicos. Entre ellos, y en ambos casos, aparece Horus, dios tutelar, vengador, garante, propiciador y heredero ${ }^{72}$ de las potencias de los dos primeros. Esto último dicho a partir de la proyección desde la esfera mitológica hacia lo terreno que sustenta la fórmula Horus sucede a Osiris $^{73}$. De la misma manera, esta fórmula es plasmada en el Libro de las Cavernas, segunda división, cuarta escena, "Oh Osiris a la cabeza de los Occidentales, cuya cueva es protegida por su hijo Horus. Oh Rey $N$ es tu hijo Horus» ${ }^{74}$. La gran trilogía divina nos permite visualizar en primera instancia una relación de transferencia que con Re como punto de partida se desplaza hacia Osiris para luego desembocar en Horus, parte de la configuración solar como heredero oficiante del Osiris que se renueva.

De esta forma se establece una compleja relación entre Re que domina y desencadena la reconversión, Osiris perviviendo allende su putrefacción y Horus que inexorablemente presencia un proceso de continuidad y ritualización de la relación de $-\mathrm{y}$ con- con su padre Osiris.

En cuanto a la referencia de Pir. §160c «este es el hijo de tu cuerpo eternamente», en el Libro de la Tierra, división A, primer registro, tercera escena, Re se manifiesta del mismo modo: "Yo protejo al que pertenece al Occidental, cuyo sitio está por delante de aquellos entre los cuales él está. Yo protegeré al Hijo del Sol, surgido de su cuerpo, Ramsés VI, el justificado. Yo llamo a los cuerpos entre los cuales él se encuentra ${ }^{75}$. Lo novedoso no es la fórmula Horus sucediendo a Osiris sino la contigüidad ontológica que supone la presencia de Re y la llegada del faraón a Osiris como $3 h$, es decir que naturalmente ello significa que él (el faraón) se ha unido con él ( $a$ Osiris) como dios en la forma de rey ${ }^{76}$. Es de esta manera como puede aceptarse lo que

70 La seguridad que significaba para Osiris la capacidad de conservar intactas sus reliquias y de este modo evitar su definitiva descomposición, y para Re el hecho de ser protegido en un ámbito eventualmente hostil, la Duat, evitando también su desintegración, constituye una dialéctica funeraria de acto-potencia (por ende su movimiento).

71 Abt y Hornung 2003, 84.

$72 \gg$ Der Vestorbene gilt daher als Erbe $(i w 3 w)$ und als Stellvertreter (stí) des Re. Da jedoch Re als unterweltlicher Sonnengot mit Osiris vereignit ist,wird der Verstorbene parallel dazu dazu zum Sohn ( $z 3)$ und Erbe (iw3w) des Osiris, d.h. er wird mit Horus geglichen, das in diesem Zusammenhang ebenfalls als Sonnengot zu verstehen ist", Barta 1985, 27.

73 Frankfort 1976, 146-161.

74 La versión jeroglífica corresponde a Piankoff 1942, pl. XXI. También en el Libro de las Puertas, segunda división, tercer registro, -versión jeroglífica que corresponde a Hornung 1979-1980, 36-Atum dice: «Mi padre Re está justificado contra ustedes, yo estoy justificado contra ustedes. Yo soy el hijo que procede de su padre, y soy el padre que procede de su hijo». Cita que manifiesta a Atum como padre e hijo del creador y regenerador supremo, conformando de este modo una teleología divina que confiere a Re el dominio de todo tiempo y espacio.

75 La versión jeroglífica corresponde a Piankoff 1953, pl. V, IX .

76 Jansen-Winkeln 1996, 210. 
aquí se sostiene acerca de la posibilidad de hallar un antecedente de la relación ReOsiris en los TdP, no como «relación de los complejos de Re y Osiris» sino como precedente de la vinculación omnipresente en los Libros del Más Allá del Reino Nuevo.

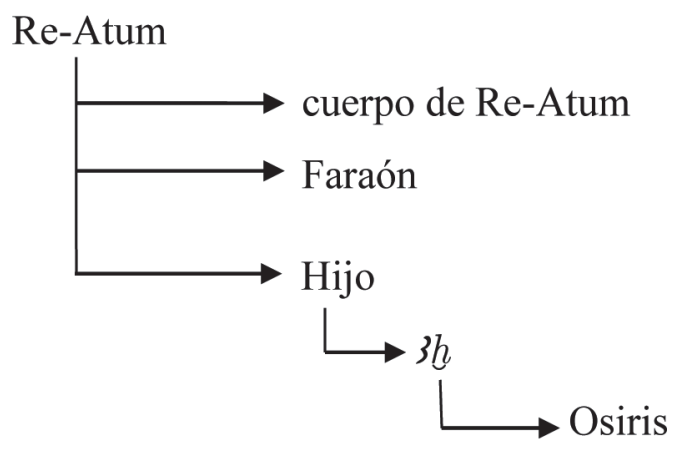

Figura 2. Relación estructurante en la liturgia TP 218

Lo que los Libros del Más Allá del Reino Nuevo expresan es cierto carácter sincrético, sintético y superador en el sentido de un reforzamiento de elementos presentes en las anteriores compilaciones funerarias.

La configuración de la transfiguración, o ser «espiritual», benéfico, glorioso tiene una estrecha vinculación con Re. El faraón, transfigurado en y con Re, se une a Osiris en un movimiento periódico de características cósmicas ${ }^{77}$.

A modo de ejemplo, en el segundo registro de la Sexta Hora del Libro del Amduat, Re pasa cerca del cadáver de Osiris y de las tumbas - hwwt- que contienen sus imágenes, devenidas en forma a su paso. Como bien marca Hornung ${ }^{78}$ estamos en presencia del momento más importante, crítico y trascendental del trayecto.

Precisamente, el nombre de una de estas mansiones o tumbas es hww dm $d y t h r w$ «tumba (o mansión) que Horus une», en clara alusión al papel mitológico de Horus acercando, mediante los rituales, a su padre fallecido con el dios solar y el dios de los difuntos.

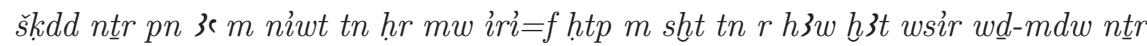
pn 3 $n$ ntrw imyw sht tn mni=f $r$ nw n hwwt št3wt hriwt sšmw ni wsir iw htr pn $\underline{d} w i=f \underline{h} r$-tp nn hwwt št3wt hww pw sdmw ntr $p n^{79}$

Este gran dios viaja por esta ciudad sobre el agua, rema en ese campo en las cercanías del cadáver de Osiris. Este gran dios da órdenes a aquellos dioses que están en los campos. El atraca junto a estas misteriosas mansiones que contienen las imágenes de Osiris.

$n s w y t=t n n=\underline{t} n$ niwswt $\check{s} z p \quad n=\underline{t} n$ h $\underline{d} \underline{t} t=\underline{t} n$ tpw $=t n^{80}$

Su realeza es suya, reyes del Alto Egipto, reciban sus coronas blancas sobre sus cabezas, están contentos, provistos con sus ofrendas.

\footnotetext{
77 En el mismo sentido, Barta 1981, 149-150.

78 Hornung 1999, 37.

79 Sexta hora del Libro del Amduat, sexta hora, segundo registro. La versión jeroglífica corresponde a Hornung $1963,106$.

80 Op. Cit., 109.
} 


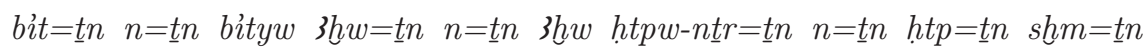
b3 $w=\underline{t} n h t p=\underline{t} n^{81}$

Sus coronas rojas pertenecen a ustedes, reyes del Bajo Egipto, sus 3 hw les pertenecen a ustedes, 3hw. Sus ofrendas divinas les pertenecen, para que puedan estar contentos.

sšt3 (misterioso, mistérico), b3 (sustancia funeraria) y $d m d w$ (unión) son las palabras claves que según Assmann ${ }^{82}$ indican la trascendencia de la unificación de los dos dioses en un solo b3. Según Barta, el rey muerto es como Re y como Osiris; él es Re como Ba, y es Osiris como cadáver ${ }^{83}$ y afirma luego que el Ba del muerto aparece como imagen o retrato $(m i-n t)$ del $\mathrm{Ba}$ del sol ${ }^{84}$, el cual no puede eludir la realidad de una sustancia eminentemente funeraria en un espacio de transferencia como la Duat -espacio general de los difuntos-. Tú eres Uno que se convierte en Dos, Tú eres Dos que se convierte en Osiris, el gran cadáver en Occidente ${ }^{85}$.

La capacidad de «trascender» revierte en Osiris y por extensión a los difuntos, al momento del encuentro con Re. De este modo la bivalencia divina funeraria es manifiesta; Osiris deviene en cadáver egregio propiciado por lo que Re dispensa. Según Barta ${ }^{86}$, la cita anterior en la que Re se dirige a Osiris trae con ello la compleja relación entre Osiris y el dios solar en una forma precisa, que está expresada en la conversación de Re a Osiris.

La continuidad sustentada por transformaciones sucesivas -en el sentido de una progresión con un comienzo y un fin que marcan una superación- obedece a la secuencia que la interacción funeraria comienza y la gestación culmina. «Ven Re... en tu nombre del vivo que viene a la existencia en la Duat $\rangle^{87}$, es el punto de partida de la interacción. Re se asume como Osiris, como difunto preclaro con quien comparte el trance de una realidad disminuida a un futuro reconvertido; en el medio, y como motoris causa, el recíproco vínculo divino.

La asunción solar de una naturaleza decreciente, corruptible, amenazada, aunque con la potencia cíclica inmanente como para regenerarse desde la inmovilidad, "¡Bienvenido! ¡Bienvenido! Después de (la) transformación. Re ha salido después de sus transformaciones. ¡Salgan, salgan, después de sus transformaciones! Re ha venido a los cielos después de sus transformaciones, al gran cielo» ${ }^{88}$, sumada a la asunción osiriana de la naturaleza solar con sus propiedades genésicas, delinean la reciprocidad funcional entre ambas divinidades; "yo (Re) doy aliento a Aquel cuyas formas están ocultas, a Osiris» ${ }^{89}$.

De este modo, lo que en TdP 218 Pir $\S 160$ c se refiere como un abrazo entre ReAtum y el difunto/Unas/Osiris, describe un punto primordial en la liturgia del faraón, probablemente el de mayor cercanía ontológica entre Re y Osiris. En los Libros del

\footnotetext{
Op. Cit., 109.

Assmann 1995, 137. Ver también Eschweiler 1994, 295-296.

Barta 1985, 24.

Op. cit.

Libro de las Cavernas, tercera división, segundo registro, quinta escena. La versión jeroglífica corresponde a Piankoff 1953, pl. XXXIII, IX.

86 Barta 1994, 60.

87 Libro del Amduat, segunda hora, tercer registro (Schlußtext). Hornung 1963, 37.

88 Libro de las Puertas, décima hora, tercer registro. La versión jeroglífica pertenece a Hornung 1979-1980, 350.

89 Libro del Amduat, primera hora, cuarto registro (Schlußtext), Hornung 1963, 18-19.
} 
Más Allá del Reino Nuevo, este encuentro es el nexo central que rige, determina y condiciona el pulso interior de los textos -aunque con las marcas particulares que cada texto le aporta $-{ }^{90}$.

\section{Conclusiones}

Los Libros del Más Allá del Reino Nuevo están constituidos según nuestro análisis por vertientes de concepciones teológicas de índole diversa que se funden en una reconfiguración ya prefijada sucinta y en ocasiones vagamente en las compilaciones funerarias más importantes.

En el caso particular estudiado, una de estas concepciones -la unión entre Re y Osiris- encuentra en la TdP 219 un verdadero antecedente que los Libros del Más Allá del Reino Nuevo enriquecen y redefinen.

En los TdP, el ciclo solar y el osiriano -en relación al culto funerario y enfatizado en la transfiguración del difunto-, suponían un límite inicial -la muerte- y uno final -la resurrección y/o reconversión-, que si bien no dejaban de reproducirse, se manifestaban como realidad inmutable. La recurrencia que implicaba el ciclo solar amenazado recreaba constantemente aquel proceso pero como presencia dinámica activa.

En esta disposición, el faraón en su devenir con Re es el actuante que obra de liberador, reproductor y catalizador de las fuerzas cíclicas. Por ello, la importancia de lo que el Más Allá alberga trasciende su dinámico hieratismo y es un elemento de primer orden a la hora de delinear la dinámica de una sociedad ${ }^{91}$. Esta dinámica se sustenta, en el contexto aquí estudiado, en la posibilidad de reutilizar un texto funerario de la realeza, o presuntamente concebido para dicho ámbito, por parte de esferas privadas no-reales.

La triple ecuación Faraón-Re-Osiris ${ }^{92}$, sustentada en el carácter identitario de la relación, implica en los Libros del Más Allá del Reino Nuevo una vuelta y un reforzamiento de la situación que los TdP iniciaron. Vuelta, en relación a la trascendencia de la suerte real vinculada al conjunto; reforzamiento, por cuanto la inclusión general de los difuntos revitaliza la función real inmanente.

90 El mito de Osiris y el mito del recorrido solar forman parte en común como tiempo lineal y tiempo cíclico, historia y cosmos, memoria y renovación. Ellos son contrarios, a los que el pensamiento egipcio no contrapone el uno contra el otro, sino que liga en una unidad mayor, Assmann 2004, 29.

91 El mundo funerario - su configuración, sus imágenes, su funcionamiento, en fin, su composición integral-, no es sino el resultado de una proyección de datos de la realidad compartidos, cuanto menos, por sus gestores. Dichos datos comprenden una variedad inmensa de elementos, desde referencias objetivas (paisajes, habitantes) hasta dispositivos de la emotividad o de la apercepción. El hecho de que ocasionalmente tanto las representaciones como los textos nos devuelvan una imagen «estilizada» que combina su connotación original una fuerte carga fantástica que la reproduce como una contra-realidad o mejor una supra-realidad asombrosa, tiene que ver precisamente con la magnitud del fenómeno que sustentan, esto es, la muerte, el devenir post-mortem y la renovación eterna. De esta forma, aquella carga de sentido proyectada, deviene en vector de una realidad trascendente que por ello no puede ser expresada en términos asequibles o aprehensibles. Lo interesante de esta proyección es que procura en cierta forma plasmar en un ámbito permanente, un ordo sociabilis que en realidad es lo pretendido para el mundo terreno y que, por estar sometido a la contingencia de lo humano, deviene en irrealizable, siempre perfectible o fragmentario.

92 Cada uno de los términos de la suprema ecuación es una en sí misma; vale decir; la complejidad del Faraón, de Re y de Osiris no se agota en su propia e intrincada «personalidad». Las variables que las numerosas relaciones establecidas delinean -muchas de las cuales se vinculan con competencias consustanciales a otras entidades-, constituyen un anexo inherente. 


\section{Referencias bibliográficas}

Th. Abt y E. Hornung, Knowledge for the Afterlife: the Egyptian Amduat-A quest for immortality. Living Human Heritage Publications, Zürich, 2003.

J. P. Allen, «Reading a Pyramid,» en C. Berger y N. Grimal (eds.), Hommages à Jean Leclant, C. Berger et al. (ed.), vol. 1, Institut français d'archéologie orientale Press, Caire, 1994.

J. P. Allen, The Ancient Egyptian Pyramid Texts, Society of Biblical Literature, Atlanta, 2005. Th. Allen, Horus in the Pyramid Texts. Chicago, 1916.

H. Altenmüller, Texte zum Begräbnisritual in den Pyramiden des Alten Reiches, Ägyptologische Abhandlungen 24, Wiesbaden, 1972.

J. Assmann, «Verklärung,» Lexikon der Ägyptologie, vol. 6, Wiesbaden, (1986) 998-1006.

J. Assmann, «Egyptian Mortuary Liturgies,» en S. Israelit-Groll (ed.), Studies in Egyptology Presented to Miriam Lichtheim, Magnes Press, vol. 1, Jerusalem, (1990) 1-45.

J. Assmann, Egyptian Solar Religion in New Kingdom. Re, Amun and the crisis of polytheism, Kegan Paul, New York, 1995.

J. Assmann, Ägyptische Geheimnisse, Wilhelm Fink Verlag, München, 2004.

J. Assmann, Death and Salvation in Ancient Egypt, Cornell University Press, 2005.

W. Barta, Die Bedeutung der Pyramidentexte für den verstorbenen König. Münchner Ägyptologische Studien 39, Deutscher Kunstverlag, München, 1981.

W. Barta, Die Bedeutung der Jenseitsbücher für den vestorbenen König. Munchner Ägyptologische Studien 42, Deutscher Kunstverlag, München, 1985.

W. Barta, Komparative Untersuchungen zu vier Unterweltsbüchern. Peter Lang, Frankfurt am Main-Bern-New York-Paris, 1994.

H. Beinlich, Die «Osirisreliquien»: Zum Motiv der Körperzergliederung in der altägyptischen Religion. Ägyptologische Abhandlungen 42, Harrassowitz Verlag Wiesbaden, 1984.

M. Broze, y A. Cywie, «Généalogie et topologie. Pratique du mythe dans la formule 219 des Textes des Pyramides», en M. Broze, Chr. Cannuyer y Fl. Doyen (eds.), Acta Orientalia Belgica 21, Bruxelles, (2008) 63-75.

E. Brovarsky, «The Doors of Heaven», Orientalia 46, Gregorian Biblical Press, Rome, (1977) 107-115.

T. DuQuesne, The Osiris-Re Conjunction with Particular Reference to the book of Dead, en B. Backes, I. Munro, y S. Stöhr (eds.). Totenbuch-Forschungen. Gesammelte Beiträge des 2. Internationalen Totenbuch-Symposium 2005, Harrassowitz Verlag Wiesbaden, (2006) 23-34.

S. Edwards, Symbolism of the Eye of Horus in the Pyramid Texts. PhD. University College of Swansea, 1995.

A. Erman y H. Grapow, Wörterbuch der Ägyptischen Sprache, Vols. 3 y 4, Akademie Verlag, Berlin, 1971.

P. Eschweiler, Bildzauber im alten Ägypten: die Verwendung von Bildern und Gegenständen in magischen Handlungen nach den textes den Mittleren und Neuen Reiches. Orbis Biblica et Orientalis 137, Vandenhoeck and Ruprecht, Fribourg-Göttingen, 1994.

R. Faulkner, The Ancient Egyptians Pyramid Texts. Oxford University Press, Oxford, 1969.

H. Frankfort, Reyes y Dioses, Revista de Occidente, Madrid, 1976.

K. Goebs, Crowns in early Egyptian Funerary Literature Royalty Rebirth and Destruction, Griffith Institute Monographs, Oxford Griffith Institute, 2008.

J. G. Griffiths, The Origins of Osiris and his Cult. Studies in the History of Religions. Supplements to NVMEN 40, E.J. Brill Leiden, 1980. 
H. Hays, y W. Schenck, «Intersection of Ritual Space and Ritual Representation: Pyramid Texts in Eighteenth Dynasty Theban Tombs», en P. Dorman y B. Bryan (eds.), Sacred Space and Sacred Function in Ancient Thebes. Oriental Institute, University of Chicago Press, Chicago, 2007.

H. Hays, «Unreading the Pyramids», Bulletin de l'Institut français d'archéologie orientale 109, Caire, (2009) 195-220.

H. Hays, «Old Kingdom Sacerdotal Texts», Jaarbericht van het Vooraziatisch-Egyptisch Genootschap «Ex Oriente Lux», Leiden, (2009a) 47-94.

H. Hays, «Unreading the Pyramids», Bulletin de l'Institut français d'archéologie orientale 109, (2009b) 195-220.

H. Hays, The Organization of the Pyramis Texts: Tipology and Disposition, Probleme der Ägyptologie 31. E. J. Brill, Leiden-Boston, 2012.

J. Hellum, «Toward an Understanding of the Use of Myth in the Pyramid Texts», Studien zur Altägyptischen Kultur 43, Buske Verlag, Hamburg, (2014) 123-142.

J. Hoffmeier, "The possible origins of the tent of purification in the Egyptian funerary cult», Studien zur Altägyptischen Kultur 9, Buske Verlag, Hamburg, (1981) 167-177.

E. Hornung, Das Amduat. Die Schrift des verborgenen Raumes, Ägyptologische Abhandlungen 7, 2 Vols. Wiesbaden. O. Harrassowitz (1963).

E. Hornung, Das Buch von den Pforten und Jenseits. 2 Vols. Geneva. Aegyptiaca Helvetica. 1979-1980.

E. Hornung, Idea into Image. Essays on Ancient Egyptians Thought, Princeton University Press, Timken Publishers, New York, 1992.

E. Hornung, The Ancient Egyptian Books of the Afterlife, Cornell University Press, Ithaca, 1999.

K. Jansen-Winkeln, «"Horizont” und "Verklärtheit”: Zur Bedeutung der Wurzel Ax», Studien zur Altägyptischen Kultur 23, Buske Verlag, Hamburg, (1996) 201-215.

J. B. Jørgensen, «Myth and cosmography: on the union of Re and Osiris in two types of religious discourse», in: Horn, Marteen et.al. (eds.), Current Research in Egyptology 2010: Proceedings of the Eleventh Annual Symposium, Oxford, (2011) 71-80.

M. Lichtheim, Ancient Egyptian Literature. Volume I: The Old and Middle Kingdoms, University of California Press, 1975.

A. Martin, Le Corps en Egypte ancienne. Enquête lexicale et anthropologique. Archaeology and Prehistory. Thèse pour obtenir le grade de Docteur. Université Paul Valéry - Montpellier III, 2013.

B. Mathieu, «Modifications de texte dans la pyramide d'Ounas», Bulletin de l'Institut français d'archéologie orientale 96, Caire, (1996) 289-311.

B. Mathieu, «Mais qui est donc Osiris? Ou la politique sous le linceul de la religion». Ègypte Nilotique et Méditerranéenne 3, Montpellier, (2010) 77-107.

B. Mathieu, Linguistique et archéologie: l'usage du déictique de proximitè ( $\mathrm{pn} / \mathrm{tn} / \mathrm{nn}$ ) dans les textes des pyramides. En Ph. Collombert, D. Lefèvre, S. Polis, y J. Winand (eds), Aere perennius: mélanges égyptologiques en 1'honneur de Pascal Vernus, Leuven: Peeters, (2016) 407-427.

F. Mauric-Barberio, «Le premier exemplaire du Livre de l'Amduat», Bulletin de l'Institut français d'archéologie orientale 101, Caire, 2001, 315-350.

M. A. Molinero Polo, «Realeza y concepción del universo en los Textos de las Pirámides», en Boletín de la Sociedad Española de Ciencias de las Religiones, vol. 12, Madrid, 1999.

A. J. Morales, «A false-door spell in the Pyramid Texts? An interpretation for the discontinuation of PT355», Studien zur Altägyptischen Kultur 45, Hamburg (2016) 237-255. 
A. J. Morales, «From voice to papyrus to wall: Verschriftung and Verschriftlichung in the Old Kingdom Pyramid Texts», en M. Hilgert (ed.), Understanding Material Text Cultures. A Multidisciplinary View, Berlin: De Gruyter, en prensa, 57-126.

M. Nuzzolo, «Royal architecture and Pyramid Texts: Some remarks on 'Kingship' in the III Millennium B.C.», Recent Discoveries and Latest Researches in Egyptology, Proceedings of the First Neapolitan Congress of Egyptology, Naples, (2010) 177-197.

J. Osing, «Zur Disposition der Pyramidentexte des Unas», Mitteilungen des Deutschen Archäologischen Instituts Abteilung Kairo 42, Wiesbaden, (1986) 131-144.

L. Pantalacci, «A propos de reliques osiriennes. Review of H. Beinlich, Die "Osirisreliquien”: zum Motiv der Körperzergliederung», Chronique d'Égypte 62 (1987) 108-123.

Piankoff, A. «Le livre des Quererts. Seconde division - Cinquième division», Bulletin de l'Institut français d'archéologie orientale 42, Caire 1-62, 1942.

A. Piankoff, (1953). La Creation du disque Solaire. Le Caire. Biblothèque d'Ètude Institut français d'archéologie orientale $\mathrm{N}^{\circ}$ 19. Le Caire. 1953.

A. Piankoff, The Pyramid of Unas, Princeton University Press, Princeton 1968.

G. Pinch, Handbook of Egyptian Mythology, ABC-CLIO, California and Oxford, 2002.

J. Quack, «Ein Prätext und seine Realisierung. Facetten des Ägyptischen Mundöffnungsritual», en Dücker y Roeder (eds.), Text und Ritual. Kulturwissenschaftliche Essays und Analyses von Sesostris bis Dada, Heidelberg, (2005) 165-185.

C. Reintges, «The oral-compositional form of Pyramid Text discourse», en F. Hagen et alii (eds.), Narratives of Egypt and the Ancient Near East. Literary and Linguistic Approaches, Orientalia Lovaniensia Analecta 189, Leuven, Peeters, (2011), 3-54

Schott, S. Mythe und Mythebildun im alten Äegypten, Untersuchungen zur Geschichte und Altertumskunde Ägyptens 15, Leipzig, 1945.

K. Sethe, Die altagyptische Pyramidentexten, J. C. Hinrichs, Lepzig, 1908-1922.

T. Shmakov, Critical analysis of J. P. Allen's The Ancient Egyptian Pyramid Texts. A. K. Eyma (ed.), Omsk-Tricht, 2012.

M. Smith, «Osiris NN or Osiris of NN?», en B. Backes, I. Munro, y S. Stöhr, (eds.) Totenbuch-Forschungen. Gesammelte Beiträge des 2. Internationalen Totenbuch-Symposiums 2005. Studien zum Altägyptischen Totenbuch 11. Wiesbaden, (2006) 325-337.

M. Smith, «Religion de l'Égypte ancienne», Conférences de l'année 2012-2013. Annuaire de l'École pratique des hautes études, Section des sciences religieuses, (2013) 87-101.

J. Spiegel, «Das Auferstehungsritual der Unas -Pyramide», Ägyptologische Abhandlungen 23, Wiesbaden, 1971.

F. von Känel, «Selqet», Lexikon der Ägyptologie, vol. 5, Wiesbaden, (1983) 830-833. 